\title{
A Model for Shear Stress Relaxation around a Fiber Break in Unidirectional Metal Matrix Composites*
}

\author{
Nobutada $\mathrm{OHNO}^{* *}$ and Taichiro YAMAKAWA**
}

\begin{abstract}
A model is presented to have insights into the shear stress relaxation around a fiber break in unidirectional metal matrix composites reinforced with long brittle fibers. A cylindrical cell containing a broken fiber is considered, and a bilinear approximation of the fiber stress distribution in the broken fiber is employed to derive a simple differential equation for the shear stress relaxation. The resulting relaxation equation is applied to the cell subjected to either constant or increasing strain. It is thus shown that the shear stress relaxes very slowly in comparison with the axial normal stress in the matrix, and that the analytical solution obtained in the case of constant strain agrees well with the numerical analysis performed by Du and McMeeking. It is also shown that the relaxation equation under constant strain is almost derivable on the basis of the overall balance of energy in the cell. In addition effect of the radial gradient of shear stress in the matrix is discussed.
\end{abstract}

Key Words: FRM, Stress Relaxation, Creep, Long Brittle Fiber, Fiber Break, Interfacial Shear Stress

\section{Introduction}

Creep behavior of unidirectional metal matrix composites reinforced with long brittle fibers, typical of which are silicon carbide SCS-6 fiber/titanium alloy composites, has been studied recently in several works. The following findings were thus obtained.

Performing creep tests of a unidirectional SCS-6/ Ti-15-3 composite at $450^{\circ} \mathrm{C}$, Ohno et al. ${ }^{(1)-(3)}$ showed experimentally that the composite may rupture in creep at stress much lower than the tensile strength although the reinforcing fibers do not creep at all at the test temperature. Similar results were reported by Schwenker et al. ${ }^{(4)}$ This creep rupture can be explained as follows: At high temperature, matrix stress is relaxed, and accordingly fiber stress is increased to cause the composite to elongate longitudinally. Then, fiber breakage may occur progressively starting with the weakest fiber; thus, if a certain number of fibers

* Received 13th June, 1996. Japanese original: Trans. Jpn. Soc. Mech. Eng., Vol. 61, No. 591, A(1995), pp. 2399-2405(Received 13th April, 1995)

** Graduate School of Engineering, Nagoya University, Chikusa ku, Nagoya 464-01, Japan are broken, overall rupture of the composite can be induced. The creep rupture may be hastened if the reinforcing fibers suffer from environmental damage $^{(4)}$. Creep tests of $\gamma$-TiAl reinforced with continuous $\mathrm{Al}_{2} \mathrm{O}_{3}$ fibers were performed in four-point flexure by Weber et al. ${ }^{(5)}$

McLean $^{(6)}$ analyzed longitudinal creep elongation of unidirectional metal matrix composites reinforced with continuous fibers. He expressed analytically the creep elongation by taking into account the load transmission, induced by matrix viscosity, from the matrix phase to the fibers. He thus predicted that the creep elongation develops until matrix stress is completely relaxed. This analysis, however, is not applicable to the creep rupture mentioned above, because he did not consider any fiber break in the modeling. He therefore extended his model using internal state variables representing damage evolution in composites $^{(7)}$.

Numerical simulations of creep of unidirectional metal matrix composites with fiber breaks were performed in recent works ${ }^{(8)-(11)}$. It was thus found that the stress recovery segments in broken fibers extend with time. This means that the interfacial shear 
stress acting on each stress recovery segment relaxes with time due to matrix viscosity. Since the extension of stress recovery segments makes the load carrying capacity of fibers decrease, it may bring about the creep rupture. To predict the creep rupture time, therefore, it is necessary to take into account the relaxation of the interfacial shear stress. Especially Du and McMeeking(11) showed, on the basis of their shear lag analysis of a unit cell, that the relaxation plays an important role in long term creep. Mason et al. ${ }^{(12)}$ analyzed stress profiles around a fiber break in a unidirectional planar composite with nonlinear matrix viscosity though limited to the case of constant applied strain.

In this paper, a simple model is presented to have insights into the relaxation of interfacial shear stress around a fiber break in unidirectional metal matrix composites. A differential equation for the relaxation of interfacial shear stress is derived by considering a cylindrical cell containing a broken fiber and by approximating bilinearly the axial stress distribution in the broken fiber. The resulting equation is applied to the cell subjected to constant strain first. Thus, characteristics in the relaxation of interfacial shear stress are discussed, giving rise to an analytical relaxation relation, which is shown to agree well with the numerical simulation done by Du and McMeeking ${ }^{(11)}$. Discussion of the relaxation equation in the context of the overall balance of energy in the cell is also given under constant strain. The relaxation equation is applied further to the cell subjected to tensile straining at constant strain rate in order to discuss effect of the strain rate induced term in the relaxation equation. Besides, effect of the radial gradient of shear stress in the matrix on the relaxation characteristics of interfacial shear stress is analyzed.

\section{Model for Relaxation of Interfacial Shear Stress}

We consider a unidirectional metal matrix composite reinforced with long brittle fibers of diameter $D$. Let the fiber volume fraction be $V_{f}$. We assume that the fibers deform only elastically while the matrix exhibits power-law creep as well, and that some fibers are broken sparsely in the composite, as shown in Fig. 1. Then, axial stress in the intact fibers, $\sigma_{f}$, and longitudinal normal stress in the matrix, $\sigma_{m}$, may distribute almost uniformly in each of them except near the fiber breaks. Hence, overall longitudinal strain of the composite, $\varepsilon$, can be expressed as

$$
\begin{aligned}
& \varepsilon=\frac{\sigma_{f}}{E_{f}}, \\
& \dot{\varepsilon}=\frac{\dot{\sigma}_{m}}{E_{m}}+B \sigma_{m}^{n},
\end{aligned}
$$

where $E_{f}$ and $E_{m}$ denote Young's moduli of the fibers and matrix, respectively, $B$ and $n$ are creep constants, and $\left({ }^{\circ}\right)$ indicates the differentiation with respect to time $t$. It is noticed that the above equations can be appropriate only if the fiber break spacing is long (see Song et al. $\left.{ }^{(13)}\right)$.

Axial stress in the broken fibers, $\sigma_{f}^{b}$, changes greatly in the vicinity of each fiber break. Figure 2 illustrates the distribution of $\sigma_{f}^{b}$ in a broken fiber; $\sigma_{f}^{b}$ is zero at the fiber break, but due to the interfacial shear stress $\tau$ acting on the stress recovery segment, $\sigma_{f}^{b}$ increases to the stress level in the intact fibers, $\sigma_{f}(=$ $E_{f} \varepsilon$ ), with the increase of the distance $z$ measured from the fiber break. Let us approximate bilinearly the distribution of $\sigma_{f}^{b}$, as shown by the dashed line in Fig. 2. Then, since $\tau$ becomes constant on the stress recovery part $0 \leq z \leq \delta$, the distribution of $\sigma_{f}^{b}$ and the stress recovery length $\delta$ are expressed in the following forms, respectively, which were derived for short fibers by Kelly and Tyson ${ }^{(14)}$ :

$$
\begin{aligned}
& \sigma_{f}^{b}= \begin{cases}4 \tau z / D, & 0 \leq z \leq \delta, \\
E_{f} \varepsilon, & \delta<z,\end{cases} \\
& \delta=\frac{E_{f} \varepsilon D}{4 \tau} .
\end{aligned}
$$

The numerical analysis of $\mathrm{Du}$ and McMeeking ${ }^{(11)}$ supports the bilinear approximation of $\sigma_{f}^{b}$ mentioned

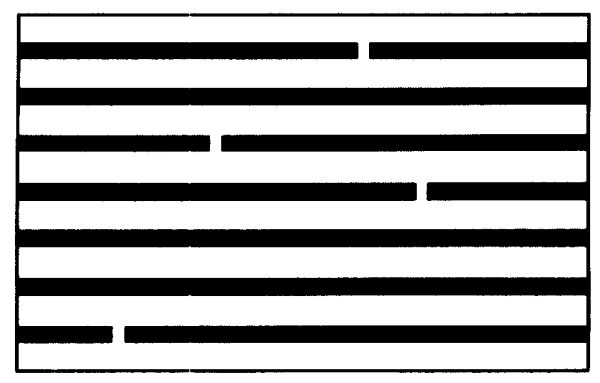

Fig. 1 Continuous fber reinforced unidirectional compos. ite with fiber breaks

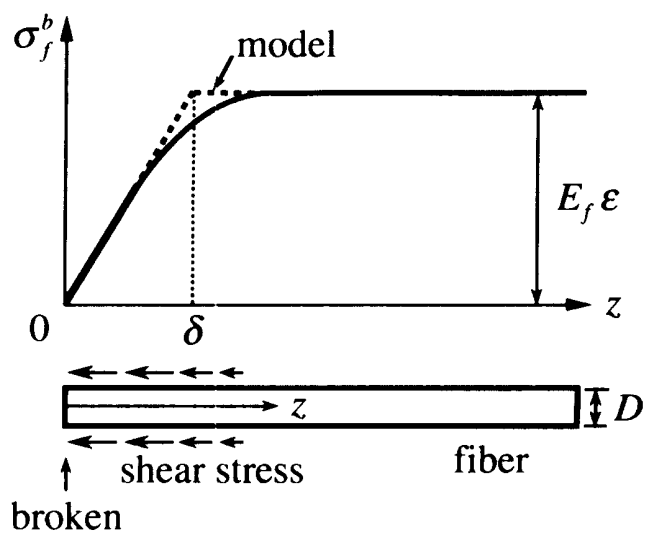

Fig. 2 Axial stress distribution in a broken fiber embedded in matrix 
above. They performed finite difference analysis based on a shear lag model to study the influence of matrix viscosity on the distribution of $\sigma_{f}^{b}$. The distribution of $\sigma_{f}^{b}$ computed by them is well approximated bilinearly even when matrix viscosity makes $\sigma_{f}^{b}$ relax to a considerable extent. Moreover, fairly uniform distribution of shear stress on the stress recovery segment is seen in the finite element creep analysis done by Kelly and Barbero ${ }^{(10)}$. Hence, the bilinear approximation of $\sigma_{f}^{b}$ is considered to be appropriate at least to first approximation.

Now, in order to derive a relaxation equation of $\tau$, let us consider a cylindrical cell in which a broken fiber is embedded (Fig. 3). The cell is infinitely long in the axial direction. Since the fiber volume fraction is $V_{f}$ in the composite, the matrix phase in the cell has the thickness

$$
w=D\left(\alpha V_{f}^{-1 / 2}-1\right) \text {, }
$$

where $\alpha=(\pi / 2 \sqrt{3})^{1 / 2}$. Here it is noticed that $w$ is taken to be the spacing between fiber surfaces since the neighboring fibers experience nearly the composite strain $^{(11)}$. We thus assume that axial strain, which is equal to the composite strain $\varepsilon$, distributes uniformly on the lateral surface of the cell. Then, the fiber break at $z=0$ has the following axial displacement relative to the lateral surface of the cell :

$$
u=\int_{0}^{\delta}\left(\varepsilon-\frac{\sigma_{f}^{b}}{E_{f}}\right) d z
$$

Substitution of Eqs. (3) and (4) into this equation gives

$$
u=\frac{D E_{f} \varepsilon^{2}}{8 \tau}
$$

This relative displacement should be compatible with the matrix shear deformation at $z=0$. If we neglect the radial gradient of shear stress in the matrix phase in the cell, and if we are based on the shear lag analysis for creep of short fiber reinforced composites $^{(15)}, \quad \dot{u}$ is expressed as

$$
\dot{u}=w \dot{\gamma}_{m}
$$

Here $\dot{\gamma}_{m}$, denoting the shear strain rate in the matrix, can be written in the following form by neglecting the elastic part:

$$
\dot{\gamma}_{m}=3 B \sigma_{e}^{n-1} \tau,
$$

where $\sigma_{e}$ represents the Mises-type equivalent stress, i.e.,

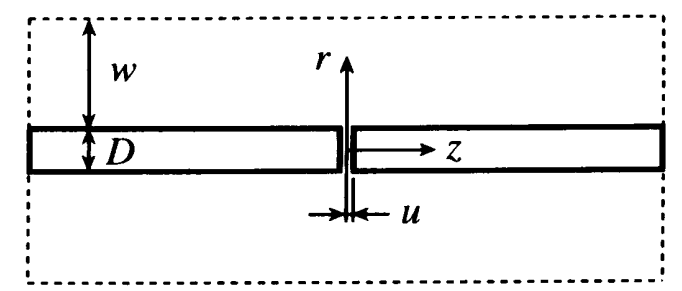

Fig. 3 Cylindrical cell containing a broken fiber

$$
\sigma_{e}=\left(\sigma_{m}^{2}+3 \tau^{2}\right)^{1 / 2}
$$

Then, differentiating Eq. $(7)$ with respect to $t$, and equating the resulting equation with Eq. ( 8 ), we have

$$
\dot{\tau}=\frac{2 \tau \dot{\varepsilon}}{\varepsilon}-\frac{24 B\left(\alpha V_{f}^{-1 / 2}-1\right) \sigma_{e}^{n-1} \tau^{3}}{E_{f} \varepsilon^{2}} .
$$

This equation represents the change of $\tau$. The first term in the right hand side is a consequence of $\dot{\varepsilon}$ : The change of $\varepsilon$ causes the stress recovery part in the broken fiber to move relatively to the lateral surface of the cell, as seen from Eq. ( 7); then, the matrix phase resists the relative movement of the stress recovery part, so that $\dot{\tau}$ is induced if sliding does not occur at the interface. The second term, on the other hand, expresses the relaxation of $\tau$ resulting from matrix viscosity.

It is noticed that Eq. ( 7 ) represents the broken fiber displacement at $z=0$, and that only the displacement compatibility at $z=0$ was imposed in deriving Eq. (11). Hence, it is the change of $\tau$ at $z=0$ that Eq. (11) expresses. It will be however shown later that the change of $\tau$ by Eq. (11) almost satisfies the overall balance of energy in the cell subjected to constant strain.

In the present work, we suppose that $\dot{\varepsilon} \geq 0$ and consequently $\tau \geq 0$. Moreover, we assume that $\tau$ is equal to the interfacial friction stress $\tau_{0}$ just after the fiber has been broken, and that $\tau$ does not exceed $\tau_{0}$. Equation (11) is then modified as

$$
i=\left\{\begin{array}{l}
\zeta, \tau<\tau_{0} \text { or } \zeta<0 \\
0, \tau=\tau_{0} \text { and } \zeta \geq 0
\end{array}\right.
$$

where

$$
\zeta=\frac{2 \tau \dot{\varepsilon}}{\varepsilon}-\frac{24 B\left(\alpha V_{f}^{-1 / 2}-1\right) \sigma_{e}^{n-1} \tau^{3}}{E_{f} \varepsilon^{3}} .
$$

Equations (12) and (13) are nondimensionalized as Eqs. (A 2) and (A 3) in the Appendix, in which the following nondimensional quantities are used:

$$
\hat{t}=B E_{f} \sigma_{c}^{n-1} t, \hat{\varepsilon}=\frac{E_{f} \varepsilon}{\sigma_{c}}, \sigma_{m}=\frac{\sigma_{m}}{\sigma_{c}}, \bar{\tau}=\frac{\tau}{\sigma_{c}},
$$

where $\sigma_{c}$ denotes an appropriate stress for the nondimensionalization. All the calculation in the present work was done by use of the above nondimensional quantities (see Appendix).

\section{Cell Subjected to Constant Strain}

\subsection{Relaxation characteristics of $\tau$ and $\sigma_{m}$}

Here, we discuss the relaxation characteristics of $\tau$ and $\sigma_{m}$ by applying Eq. (12) as well as Eq. (2) to the cylindrical cell subjected to strain holding after $t=0$, i.e.,

$$
\varepsilon(t)=H(t) \varepsilon_{0},
$$

where $H(t)$ indicates Heaviside's step function, and $\varepsilon_{0}$ is constant. We suppose that the fiber breakage in the cell occurs when the cell is strained at $t=0$. 
The change of $\sigma_{m}$ is expressed using Eq. (2) as follows:

$$
\dot{\sigma}_{m}=E_{m}\left(\dot{\varepsilon}-B \sigma_{m}^{n}\right)
$$

During the strain holding, $\dot{\varepsilon}=0$. Hence this equation, together with the initial condition $\sigma_{m}(0)=E_{m} \varepsilon_{o}$, gives

$$
\sigma_{m}(t)=\left[\left(E_{m} \varepsilon_{0}\right)^{-n+1}+(n-1) B E_{m} t\right]^{-1 /(n-1)} .
$$

On the other hand, the change of $\tau$ is expressed by Eqs. (12) and (13) with $\dot{\varepsilon}=0$ and $\varepsilon=\varepsilon_{0}$ :

$$
\dot{\tau}=-\frac{24 B\left(\alpha V_{f}^{-1 / 2}-1\right)\left(\sigma_{m}^{2}+3 \tau^{2}\right)^{(n-1) / 2} \tau^{3}}{E_{f} \varepsilon_{0}^{2}},
$$

where $\tau(0)=\tau_{0}$. It is easy to compute $\tau(t)$ by integrating numerically this equation into which Eq. (17) is substituted.

Figure 4 shows the relaxation curves $\sigma_{m}(t)$ and $\tau(t)$ calculated using Eqs. (17) and (18), respectively, with the constants given in the figure caption. It is seen from the figure that $\tau$ relaxes very slowly in comparison with $\sigma_{m}$. Let us discuss this significant feature in detail by taking the ratio of $\dot{\sigma}_{m}$ and $\dot{\tau}$ expressed by Eq. (16) with $\dot{\varepsilon}=0$ and Eq. (18), respectively:

$$
\frac{\dot{\sigma}_{m}}{\dot{\tau}}=C \frac{\sigma_{m}^{n}\left(E_{f} \varepsilon_{0}\right)^{2}}{\left(\sigma_{m}^{2}+3 \tau^{2}\right)^{(n-1) / 2} \tau^{3}},
$$

where $C=E_{m} /\left[24\left(\alpha V_{f}^{-1 / 2}-1\right) E_{f}\right]$. Now we suppose that the initial value of $\tau$ is much smaller than the intact fiber stress $E_{f} \varepsilon_{0}$ and the initial value of $\sigma_{m}$, i.e., $\tau_{0} \ll E_{f} \varepsilon_{0}$ and $\tau_{0} \ll E_{m} \varepsilon_{0}$. Then, we see from Eq. (19) that

$$
\frac{\dot{\sigma}_{m}(0)}{\dot{\tau}(0)} \approx C \frac{E_{m} \varepsilon_{0}\left(E_{f} \varepsilon_{0}\right)^{2}}{\tau_{0}^{3}} \gg 1 \text {. }
$$

This equation suggests that $\sigma_{m}$ relaxes much more quickly than $\tau$; in other words, $\sigma_{m}$ may be negligibly small during the relaxation of $\tau$.

On the basis of the above discussion, let us neglect any influence of $\sigma_{m}$ on the relaxation of $\tau$. Then, Eq.

(18) becomes

$$
\dot{\tau}=-\frac{8 \cdot 3^{(n+1) / 2} B\left(\alpha V_{f}^{-1 / 2}-1\right) \tau^{n+2}}{E_{f} \varepsilon_{0}^{2}} .
$$

Integration of Eq. (21) gives an analytical expression

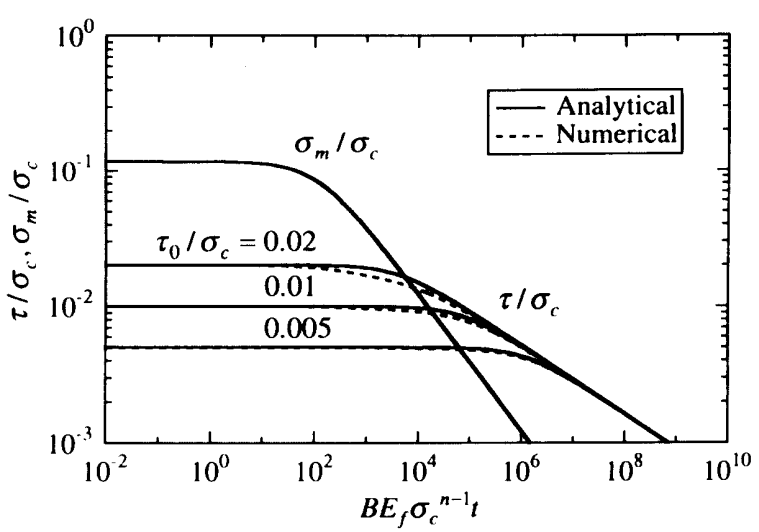

Fig. 4 Variations of $\sigma_{m}$ and $\tau$ under strain holding ( $n=3$, $E_{f} / E_{m}=3, V_{f}=0.35, E_{f} \varepsilon_{0} / \sigma_{c}=0.35$ ) of $\tau(t)$

$$
\tau(t)=\tau_{0}\left[1+(n+1) \frac{t}{t_{\tau}}\right]^{-1 /(n+1)},
$$

where $t_{\tau}$ denotes the relaxation time defined as $t_{\tau}=$ $-\tau_{0} / \dot{\tau}(0)$, i.e.,

$$
t_{\tau}=\frac{E_{f} \varepsilon_{0}^{2}}{8 \cdot 3^{(n+1) / 2} B\left(\alpha V_{f}^{-1 / 2}-1\right) \tau_{0}^{n+1}} .
$$

The change of $\tau$ expressed by Eq. (22) is indicated by the solid lines in Fig. 4. Since they are close to the dotted lines representing the numerical results of Eq. (18), we can say that $\sigma_{m}$ has negligible influence on the relaxation of $\tau$.

Substitution of Eq. (22) into Eq. (4) gives

$$
\delta(t)=\frac{E_{f} \varepsilon_{0} D}{4 \tau_{0}}\left[1+(n+1) \frac{t}{t_{\tau}}\right]^{1 /(n+1)} .
$$

Let us compare this analytical result with the numerical one obtained by $\mathrm{Du}$ and McMeeking ${ }^{(11)}$. They studied numerically how the axial stress distribution in a broken fiber in long brittle fiber reinforced unidirectional metal matrix composites varies with time at high temperature; i.e., they solved a partial differential equation of order 3 based on a shear lag theory using a finite difference method. In their analysis $\tau$ was a function of $z$ and $t$, while $\tau$ is uniform in the region of $0 \leq i \leq \delta$ in the present model. The solid circles in Fig. 5 indicate the variation of $\delta$ based on their numerical analysis. This $\delta(t)$ was determined by approximating bilinearly the axial distribution $\sigma_{f}^{b}(z, t)$ which they computed. It is noticed that they had the distribution $\sigma_{f}^{b}(z, t)$ approximated bilinearly very well. As seen from Fig. 5, the present analysis has a good agreement with their numerical analysis. Hence, we can say that the present model works well though it is a simple model.

Equation (24) with Eq. (23) predicts that $\delta$ is proportional to $t^{1 /(n+1)} \varepsilon_{0}^{(n-1) /(n+1)}$ when $t \gg t_{\tau}$. It is of interest to notice that this proportionality was derived in a rigorous but sophisticated analysis by Mason et

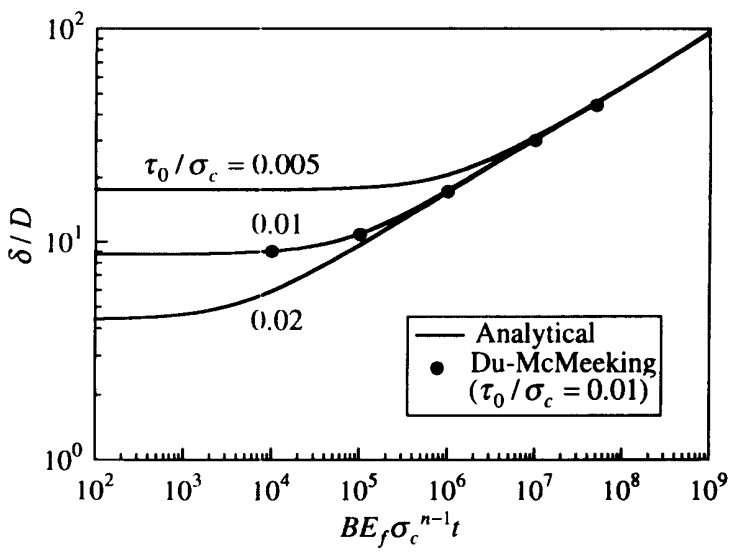

Fig. 5 Change of the stress recovery length $\delta$ under strain holding $\left(n=3, V_{f}=0.35, E_{f} \varepsilon_{0} / \sigma_{c}=0.35\right)$ 
al. ${ }^{(12)}$ for unidirectional planar composites subjected to constant strain.

\section{2 Discussion based on the overall balance of energy}

Let us remember that the relaxation equation (12) was derived by imposing the displacement compatibility at $z=0$. Since this requirement is very local, it is worth while discussing the relaxation equation on the basis of the overall balance of energy in the cell subjected to constant strain $\varepsilon_{0}$.

When $\sigma_{m}$ is completely relaxed under the relaxation condition (15), the energy dissipation rate induced by $\dot{\gamma}_{m}$ in the matrix in the cell is balanced with the change of the elastic energy stored in the broken fiber:

$$
\dot{\gamma}_{m} \tau A_{m} \delta+\frac{d}{d t} \int_{0}^{l} \frac{\left(\sigma_{f}^{b}\right)^{2}}{2 E_{f}} A_{f} d z=0,
$$

where $A_{m}$ and $A_{f}$ indicate the cross areas of the fiber and matrix parts in the cell, respectively, and $l$ is half the axial length of the cell. Substitution of Eqs. ( 3 ) and (4) into Eq. (25) and use of $A_{m} / A_{f}=(1+2 w / D)^{2}$ -1 with Eq. ( 5 ) give

$$
\dot{\tau}=-\frac{4 \cdot 3^{(n+3) / 2} B \alpha V_{f}^{-1 / 2}\left(\alpha V_{f}^{-1 / 2}-1\right) \tau^{n+2}}{E_{f} \varepsilon_{0}^{2}}
$$

From this equation, we have

$$
\tau(t)=\tau_{0}\left[1+(n+1) \frac{t}{t_{\tau}^{\prime}}\right]^{-1 /(n+1)},
$$

where

$$
t_{\tau}^{\prime}=\frac{E_{f} \varepsilon_{0}^{2}}{4 \cdot 3^{(n+3) / 2} B \alpha V_{f}^{-1 / 2}\left(\alpha V_{f}^{-1 / 2}-1\right) \tau_{0}^{n+1}} .
$$

Equations (22) and (27) are the same except for the relaxation times $t_{\tau}$ and $t_{\tau}^{\prime}$, which have the ratio

$$
\frac{t_{t}^{\prime}}{t_{\tau}}=\frac{2 V_{f}^{1 / 2}}{3 \alpha} .
$$

As seen from Fig. $6, t_{\tau}^{\prime} / t_{\tau} \approx 0.4$ if $V_{f}>0.2$. Hence, Eq. (22) differs from Eq. (27) by the time factor of about

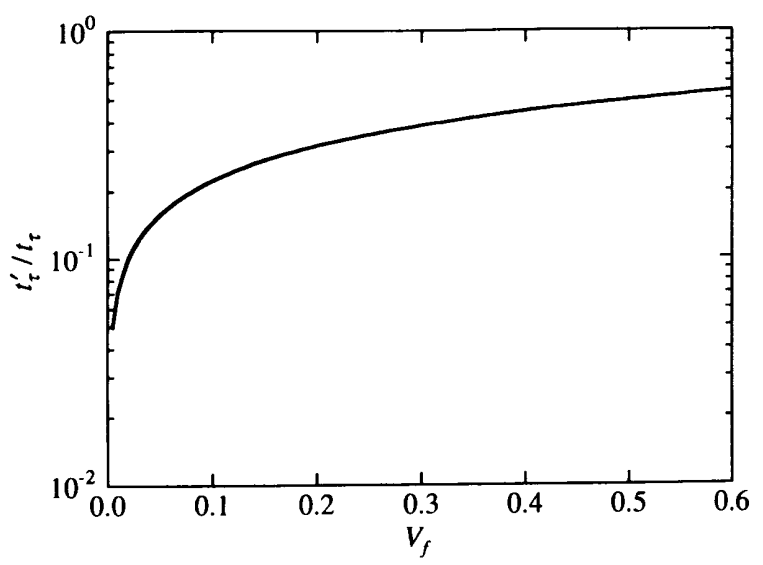

Fig. 6 Ratio of the relaxation time based on overall energy balance to that based on axial displacement compatibility at $z=0$ two. This time factor is very small in the logarithmic time scale used in Figs. 4 and 5. Therefore, we can say that under the strain holding condition (15) the overall energy balance and the axial displacement compatibility at $z=0$ led to nearly the same relaxation equation of $\tau$; in other words, the change of $\tau$ expressed by Eqs. (12) and (13) almost satisfies the overall balance of energy in the cell under constant strain.

\section{Cell Subjected to Tensile Straining}

The term $2 \tau \dot{\varepsilon} / \varepsilon$ in Eq. (13) disappeared in the previous section, because we dealt with the cell subjected to constant strain. This term represents the change of $\tau$ resulting from the increase of $\varepsilon$ (Sec. 1). In this section, we discuss effects of the term by analyzing numerically the cell subjected to tensile straining at constant strain rate following the instantaneous straining to $\varepsilon_{0}$ at $t=0$ (Fig. 7). We specify the constant strain rate to be

$$
\dot{\varepsilon}=\omega / t_{\tau},
$$

where $t_{\tau}$ denotes the relaxation time given by Eq. (23). Let us assume again that the fiber breakage in the cell occurs when the cell is strained instantaneously at $t=$ 0.

Figures $8(\mathrm{a})$ and (b) show the relaxation curves $\sigma_{m}(t)$ and $\tau(t)$ calculated for the tensile straining with the constants given in the figure caption. They were computed by integrating Eqs. (12) and (16) nondimensionalized with Eq. (14), i.e., Eqs. (A 2) and (A 1) in the Appendix. The computation was carried out for some values of the nondimensional quantity $\bar{\omega}\left(=E_{f} \omega / \sigma_{c}\right)$ until the nondimensional strain $\bar{\varepsilon}(=$ $\left.E_{f} \varepsilon / \sigma_{c}\right)$ attained 1.5 .

It is seen from Figs. 8( a ) and (b) that $\sigma_{m}$ relaxes more quickly than $\tau$ despite the increase of $\varepsilon$ induced by the tensile straining; even in the case of $\hat{\omega}$ $=0.1, \sigma_{m}$ is in the steady state when $\tau$ starts decreasing markedly. Hence, we may assume that $\sigma_{m}$ has negligible influence on the relaxation of $\tau$ under the tensile straining. Then, Eq. (13) can be approximated

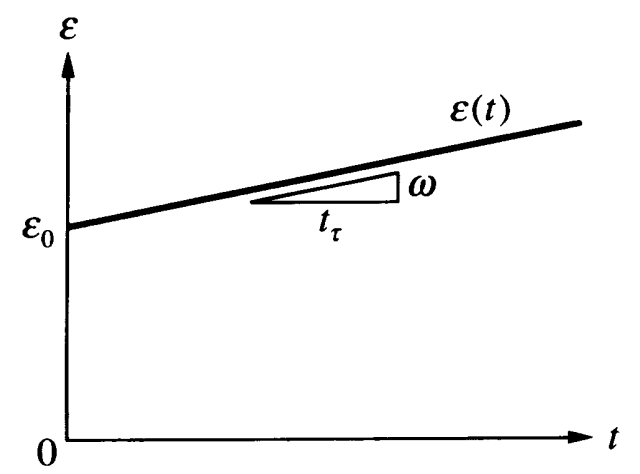

Fig. 7 Tensile straining at constant strain rate following instantaneous straining 

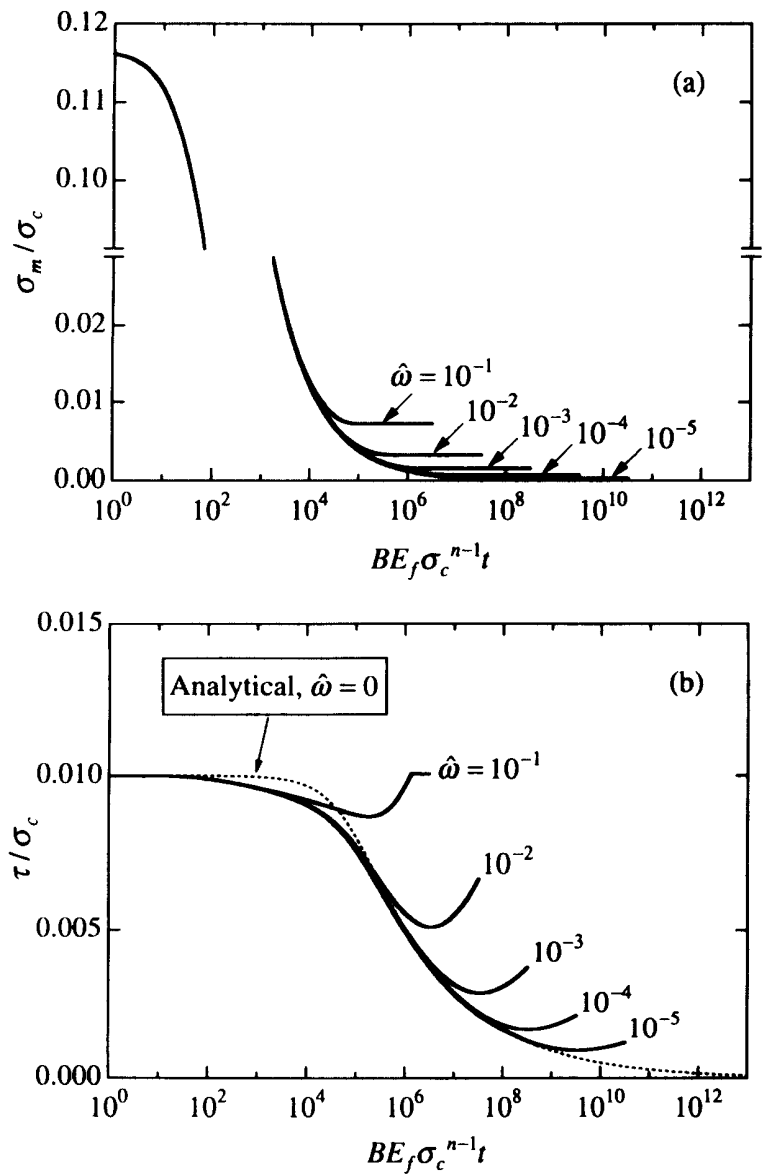

Fig. 8 Variations of $\sigma_{m}$ and $\tau$ under tensile straining at constant strain rate following instantaneous straining $\left(n=3, E_{f} / E_{m}=3, V_{f}=0.35, \tau_{0} / \sigma_{c}=0.01\right.$, $E_{f} \varepsilon_{0} / \sigma_{c}=0.35$ ); (a) $\sigma_{m}$, and (b) $\tau$, in which the dashed line indicates the relaxation curve of $\tau$ under constant strain, Eq. (22)

as

$$
\zeta=\frac{2 \tau \dot{\varepsilon}}{\varepsilon}-\frac{8 \cdot 3^{(n+1) / 2} B\left(\alpha V_{f}^{-1 / 2}-1\right) \tau^{n+2}}{E_{f} \varepsilon^{2}} .
$$

It is also seen from Fig. 8( b) that the relaxation of $\tau$ becomes more significant with the decrease of $\omega$, and that when $\omega$ is sufficiently small $\tau(t)$ is represented well by the analytical relaxation relation (22), which is valid under constant strain. These are consequences of the term $2 \tau \dot{\varepsilon} / \varepsilon$ in Eq. (13), which has less contribution to $\dot{\tau}$ with the decrease of $\dot{\varepsilon}$ and becomes zero when $\dot{\varepsilon}=0$. Thus we may say that the analytical relation (22), which was obtained under the strain holding condition in the previous section, is applicable to long term creep at low stress as well because $\dot{\varepsilon}$ can be very low in such creep.

In addition it is of interest to notice in Fig. 8(b) that $\tau$ increases after the relaxation when $\omega>0$. This is because of $\varepsilon^{2}$ in the denominator in the second term in the right hand side in Eq. (13). The first term $2 \tau \dot{\varepsilon} / \varepsilon$ on the other hand is inversely proportional to $\varepsilon$. With the increase of $\varepsilon$, therefore, the second term becomes weaker than the first term.

\section{Effect of Radial Gradient of Shear Stress in Matrix}

In the previous sections we neglected for simplicity the radial gradient of shear stress in the matrix in the cell, as seen from Eq. ( 8 ). In this section we discuss effect of the radial gradient on the relaxation time of interfacial shear stress.

Let us take account of the radial gradient of shear stress around the fiber break in the cell, i.e., $\tau=\tau(r, t)$. The axial normal stress in the matrix, on the other hand, remains to be regarded as uniform, i.e., $\sigma_{m}=$ $\sigma_{m}(t)$. Then, the equilibrium of force in the axial direction allows us to write

$$
\tau(r, t)=\frac{D}{2 r} \tau_{i}(t)
$$

where $\tau_{i}$ denotes the shear stress at the interface. Moreover, the axial displacement rate of the broken fiber end, which is given by Eq. ( 7 ) with $\tau$ replaced by $\tau_{i}$, should be compatible with

$$
\dot{u}=\int_{r_{i}}^{r_{0}} \dot{\gamma}_{m}(r, t) d r,
$$

where $r_{i}=D / 2, r_{0}=D / 2+w$, and

$$
\dot{\gamma}_{m}(r, t)=3 B\left[\sigma_{m}(t)^{2}+3 \tau(r, t)^{2}\right]^{(n-1) / 2} \tau(r, t) .
$$

Now we assume that $\sigma_{m}(t)$ has negligible influence on the relaxation of $\tau(r, t)$, as discussed already in the case of $\tau=\tau(t)$. Then, substituting Eq. (34) with $\sigma_{m}(t)$ neglected into Eq. (33), and using Eq. (32), we have

$$
\dot{u}=3^{(n+1) / 2} B \int_{r_{i}}^{r_{0}}\left[\frac{D}{2 r} \tau_{i}(t)\right]^{n} d r .
$$

When $n \neq 1$, this equation, together with Eq. (5), yields

$$
\dot{u}=\frac{3^{(n+1) / 2} B D}{2(n-1)}\left\{1-\left(2 \alpha V_{f}^{-1 / 2}-1\right)^{1-n}\right\} \tau_{i}(t)^{n} .
$$

Equation ( 7 ) with $\tau$ replaced by $\tau_{i}$ is substituted into Eq. (36 a) to derive

$$
\begin{aligned}
\dot{\tau}_{i} & =\frac{2 \tau_{i} \dot{\varepsilon}}{\varepsilon} \\
& -\frac{\left.4 \cdot 3^{(n+1) / 2} B \cdot 1-\left(2 \alpha V_{f}^{-1 / 2}-1\right)^{1-n}\right\} \tau_{i}^{n+2}}{(n-1) E_{f} \varepsilon_{0}^{2}} .
\end{aligned}
$$

For the strain holding condition (15), Eq. (37.a) is integrated analytically:

$$
\tau_{i}(t)=\tau_{0}\left[1+(n+1) \frac{t}{t_{\tau}^{*}}\right]^{-1 /(n+1)},
$$

where

$$
t_{\tau}^{*}=\frac{(n-1) E_{f} \varepsilon_{0}^{2}}{4 \cdot 3^{(n+1) / 2} B\left\{1-\left(2 \alpha V_{f}^{-1 / 2}-1\right)^{1-n}\right\} \tau_{0}^{n+1} .}
$$

When $n=1$, Eqs. (36 a) to (39 a) take the following forms:

$$
\dot{u}=\frac{3}{2} B D \ln \left(2 \alpha V_{f}^{-1 / 2}-1\right) \cdot \tau_{i}(t),
$$




$$
\begin{aligned}
& \dot{\tau}_{i}=\frac{2 \tau_{i} \dot{\varepsilon}}{\varepsilon}-\frac{12 B \ln \left(2 \alpha V_{f}^{-1 / 2}-1\right) \cdot \tau_{i}^{3}}{E_{f} \varepsilon_{0}^{2}}, \\
& \tau_{i}(t)=\tau_{0}\left[1+\frac{2 t}{t_{\tau}^{*}}\right]^{-1 / 2}, \\
& t_{\tau}^{*}=\frac{E_{f} \varepsilon_{0}^{2}}{12 B \ln \left(2 \alpha V_{f}^{-1 / 2}-1\right) \cdot \tau_{0}^{2}} .
\end{aligned}
$$

The relaxation relations (22) and (38) derived in the previous and present sections are the same except for the relaxation times $t_{\tau}$ and $t_{\tau}^{*}$. Hence, the radial gradient of $\tau$ in the matrix has influence on the relaxation behavior through the relaxation times. Figure 9 shows a comparison between them. Let us point out in this figure that $t_{\tau}^{*}$ does not have so marked dependence on $V_{f}$ as $t_{r}$. This is because the radial gradient of shear stress in the matrix makes the matrix shear creep rate insignificant at $r \gg D / 2$, leading to weakening the influence of the matrix thickness $w$ on the relaxation of interfacial shear stress. Figure 10 deals with the ratio

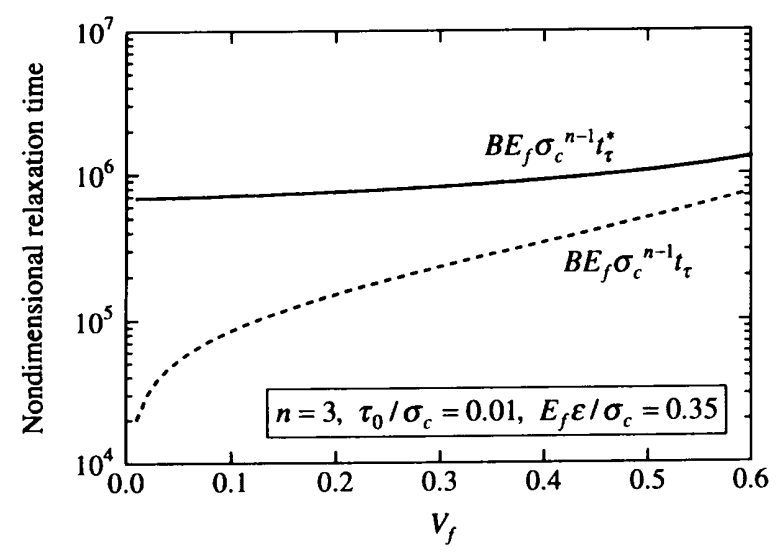

Fig. 9 Comparison between the relaxation times $t_{\tau}{ }^{*}$ and $t_{\tau}$ obtained by taking into account and neglecting the radial gradient of shear stress in matrix, respectively

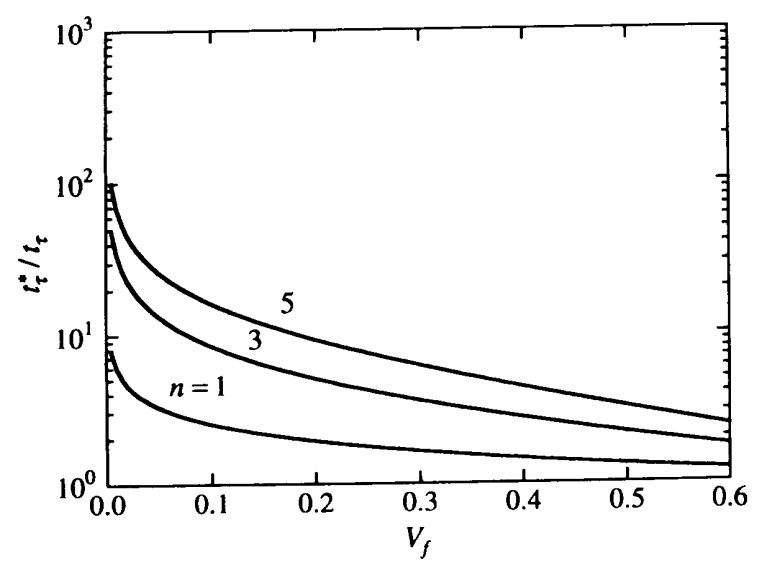

Fig. 10 Influence of the radial gradient of shear stress in matrix on the relaxation time

$$
\frac{t_{\tau}^{*}}{t_{\tau}}= \begin{cases}\frac{2(n-1)\left(\alpha V_{f}^{-1 / 2}-1\right)}{1-\left(2 \alpha V_{f}^{-1 / 2}-1\right)^{1-n},} & n \neq 1, \\ \frac{2\left(\alpha V_{f}^{-1 / 2}-1\right)}{\ln \left(2 \alpha V_{f}^{-1 / 2}-1\right)}, & n=1 .\end{cases}
$$

It is seen from the figure that $t_{\tau}^{*}>t_{\tau}$; that is, the shear stress relaxation is predicted to take place more slowly when the radial gradient is taken into account. This is because when the function $\tau(r, t)$ is expressed as Eq. (32) $\dot{\gamma}_{m}$ becomes smaller with the increase of $r$, resulting in suppressing the axial movement $u$ of the stress recovery segment in the broken fiber, as seen from Eq. (33); as a result, the relaxation time becomes longer. However, it is noticed in Fig. 10 that $t_{\tau}^{*} / t_{\tau}<10$ if $V_{f}$ is somewhat large. We remember that the relaxation of $\tau$ takes a very long time(Figs. 4, 5 and 8 ). Hence, we are allowed to say that the radial gradient of $\tau$ does not have significant influence on the relaxation behavior as far as we use such logarithmic time scales as in these figures.

\section{Conclusions}

The present work was concerned with a model which represents the relaxation of interfacial shear stress around a fiber break in unidirectional metal matrix composites reinforced with long brittle fibers.

Firstly, a simple differential equation for the shear stress relaxation was derived by assuming a cylindrical cell containing a broken fiber; in the model, the stress distribution in the broken fiber was approximated bilinearly, and the compatibility condition of axial displacement was considered at the broken fiber end. Secondly, by applying this relaxation equation to the cell subjected to constant strain, it was shown that the interfacial shear stress relaxes very slowly in comparison with the longitudinal normal stress in the matrix, and that the relaxation equation gives an analytical solution, which agrees well with the numerical analysis done by $\mathrm{Du}$ and McMeeking(11). It was also shown that the overall balance of energy in the cell under constant strain leads to nearly the same relaxation equation as that obtained by imposing the axial displacement compatibility at the broken fiber end. Thirdly, by applying further the relaxation equation to the cell subjected to tensile straining at constant strain rate, it was found that the interfacial shear stress relaxes to a certain extent depending on the strain rate, and that the analytical relaxation relation of interfacial shear stress under the strain holding condition almost holds if the strain rate is sufficiently small. Finally, the relaxation equation was modified by taking into account the radial gradient of shear stress in the matrix in the cell, and it was shown that the radial gradient does not have significant influence on the 
interfacial shear stress relaxation if the fiber volume (A.12) fraction is somewhat large.

The present model for the shear stress relaxation around a fiber break can be applied to predicting the rupture time in long term creep of unidirectional metal matrix composites reinforced with long brittle fibers $^{(16)}$.

\section{Appendix}

Here we rewrite main equations in this paper using the nondimensional quantities $\bar{t}, \bar{\varepsilon}, \bar{\sigma}_{m}$ and $\bar{\tau}$ defined by Eq. (14).

Equations (16),(12) and (13) specifying $\dot{\sigma}_{m}$ and $\dot{\tau}$ become

$$
\begin{aligned}
& \frac{d \widehat{\sigma}_{m}}{d \bar{t}}=\frac{E_{m}}{E_{f}}\left(\frac{d \hat{\varepsilon}}{d \hat{t}}-\hat{\sigma}_{m}^{n}\right), \\
& \frac{d \bar{\tau}}{d \hat{t}}=\left\{\begin{array}{l}
\bar{\zeta}, \quad \bar{\tau}<\hat{\tau}_{0} \text { or } \bar{\zeta}<0, \\
0, \quad \bar{\tau}=\hat{\tau}_{0} \text { and } \bar{\zeta} \geq 0,
\end{array}\right. \\
& \bar{\zeta}=\frac{2 \bar{\tau}}{\bar{\varepsilon}} \frac{d \bar{\varepsilon}}{d \hat{t}}-\frac{24\left(\alpha V_{f}^{-1 / 2}-1\right) \hat{\sigma}_{e}^{n-1} \hat{\tau}^{3}}{\hat{\varepsilon}^{2}},
\end{aligned}
$$

where $\widehat{\sigma}_{e}=\left(\widehat{\sigma}_{m}^{2}+3 \hat{\tau}^{2}\right)^{1 / 2}$.

Equations (17),(22) and (23), which are analytical solutions of Eqs. (16) and (12) under the strain holding condition (15), take the forms

$$
\begin{aligned}
& \bar{\sigma}_{m}=\left[\left(\frac{E_{m} \hat{\varepsilon}_{0}}{E_{f}}\right)^{-n+1}+(n-1) \frac{E_{m}}{E_{f}} \hat{t}\right]^{-1 /(n-1)}, \\
& \hat{\tau}=\bar{\tau}_{0}\left[1+(n+1) \frac{\hat{t}}{\hat{t}_{\tau}}\right]^{-1 /(n+1)} .
\end{aligned}
$$

where

$$
\hat{t}_{\tau}=\frac{\widehat{\varepsilon}_{0}^{2}}{8 \cdot 3^{(n+1) / 2}\left(\alpha V_{f}^{-1 / 2}-1\right) \widehat{\tau}_{0}^{n+1}} .
$$

The solution (27) based on the overall balance of energy in the cell, on the other hand, is rewritten as

$$
\hat{\tau}=\widehat{\tau}_{0}\left[1+(n+1) \frac{\hat{t}^{\prime}}{\hat{t}_{\tau}^{\prime}}\right]^{-1 /(n+1)},
$$

where

$$
\hat{t}_{\tau}^{\prime}=\frac{\widehat{\varepsilon}_{0}^{2}}{4 \cdot 3^{(n+3) / 2} \alpha V_{f}^{-1 / 2}\left(\alpha V_{f}^{-1 / 2}-1\right) \hat{\tau}_{0}^{n+1}} .
$$

Equation (30) for the condition of constant strain rate is nondimensionalized as

$$
\frac{d \hat{\varepsilon}}{d \hat{t}}=\frac{\widehat{\omega}}{\hat{t}_{\tau}},
$$

where

$$
\bar{\omega}=\frac{E_{f} \omega}{\sigma_{c}} .
$$

The solution (38) obtained by taking account of the radial gradient of shear stress in the matrix part in the cell takes the form

$$
\bar{\tau}_{i}=\hat{\tau}_{0}\left[1+(n+1) \frac{\hat{t}}{\hat{t}_{\tau}^{*}}\right]^{-1 /(n+1)},
$$

where

$$
\hat{t}_{\tau}^{*}= \begin{cases}\frac{(n-1) \bar{\varepsilon}_{0}^{2}}{4 \cdot 3^{(n+1) / 2}\left\{1-\left(2 \alpha V_{f}^{-1 / 2}-1\right)^{1-n}\right\} \hat{\tau}_{0}^{n+1}}, & n \neq 1 \\ \frac{\bar{\varepsilon}_{0}^{2}}{12 \ln \left(2 \alpha V_{f}^{-1 / 2}-1\right) \cdot \bar{\tau}_{0}^{2},} & n=1\end{cases}
$$

\section{References}

(1) Ohno, N., Toyoda, K., Okamoto, N., Miyake, T. and Nishide, S., Creep of Advanced Metal Matrix Composite $\mathrm{SiC}_{\mathrm{Cv}} / \mathrm{Ti}^{-15-3}$, The Processing, Properties and Applications of Metallic and Ceramic Materials, ed. by Loretto, M. H. and Beevers, C. J., Vol. I(1992), p. 447, MCE Publications.

(2) Ohno, N., Toyoda, K., Okamoto, N., Miyake, T. and Nishide, S., Creep Behavior of a Unidirectional SCS-6/Ti-15-3 Metal Matrix Composite at $450^{\circ} \mathrm{C}$, ASME J. Eng. Mater. Tech., Vol. 116, No. 2(1994), p. 208.

(3) Ohno, N., Okamoto, N., Miyake, T., Nishide, S. and Masaki, S. Jr, Acoustic Emission and Fiber Damage in Creep of a Unidirectional SCS- $6 / \mathrm{Ti}^{-}$ 15-3 Metal Matrix Composite at $450^{\circ} \mathrm{C}$, Scripta Metall. Mater., Vol. 31, No. 11(1994), p. 1549.

(4) Schwenker, S. W., Evans, D. J. and Eylon, D., Longitudinal Creep Behavior and Damage in SCS 6/Ti-6 Al-4 V Metal Matrix Composites, Tita. nium '92, Science and Technology, ed. by Froes, F. H. and Caplan, I.,(1993), p. 2593. The Minerals, Metals \& Materials Society.

(5) Weber, C. H., Yang, J. Y., Löfvander, J. P. A., Levi, C. G. and Evans, A. G., The Creep and Fracture Resistance of $\gamma$-TiAl Reinforced with $\mathrm{Al}_{2} \mathrm{O}_{3}$ Fibers, Acta Metall. Mater., Vol. 41, No. 9(1993), p. 2681.

(6) McLean, M. Creep Deformation of Metal-Matrix Composites, Compos. Sci. Tech., Vol. 23(1985), p. 37.

( 7 ) McLean, M., Mechanisms and Models of High Temperature Deformation of Composites, Mater. Res. Soc. Symp. Proc., Vol. 120(1988), p. 67.

( 8 ) Goda, K., Estimation of Creep Rupture Life of a $\mathrm{B} / \mathrm{Al}$ Composite Using Monte Carlo Simulation Method, Proc. 12th Symposium on Material and Structural Reliability, (in Japanese), (1993), p. 45, The Society of Materials Science, Japan.

(9) Barbero, E. J. and Kelly, K. W, Predicting High Temperature Ultimate Strength of Continuous Fiber Metal Matrix Composites, J. Compos. Mater., Vol. 27, No. 12(1993), p. 1214.

(10) Kelly, K. W. and Barbero, E., The Effect of Fiber Damage on the Longitudinal Creep of a CFMMC, Int. J. Solids Struct., Vol. 30, No. 24 (1993), p. 3417.

(11) Du, Z.-Z and McMeeking, R. M., Creep Models for Metal Matrix Composites with Long Brittle Fibers, J. Mech. Phys. Solids, Vol. 43, No. 5(1995), p. 701.

(12) Mason, D. D., Hui, C.- Y. and Phoenix, S. L., Stress Profiles around a Fiber Break in a Composite with a Nonlinear, Power Law Creeping Matrix, Int. J. Solids Struct., Vol. 29, No. 23(1992), p. 2829.

(13) Song, Y., Bao, G. and Hui, C. Y., On Creep of Unidirectional Fiber Composites with Fiber Damage, Acta Metall. Mater., Vol. 43, No.7(1995), p. 2615. 
(14) Kelly, A. and Tyson, W.R., Tensile Properties of Fibre-Reinforced Metals: Copper/Tungsten and Copper/Molybdenum, J. Mech. Phys. Solids, Vol. 13(1965), p. 329.

(15) Kelly, A. and Street, K. N., Creep of Discontinuous Fiber Composites, II. Theory for the Steady
State, Proc. R. Soc. Lond. A., Vol. 328(1972), p. 283.

(16) Ohno, N. and Kawabe, H., Creep Rupture Analysis of Unidirectional Metal Matrix Composites Reinforced with Long Brittle Fibers, (in preparation). 